\title{
Stewart-Treves Syndrome: A Rare But Aggressive Complication of Breast Cancer-Related Lymphedema
}

\author{
(D) Pınar Borman ${ }^{1}$, (1) Ayşegül Yaman², (1) Özay Gököz ${ }^{3}$ \\ ${ }^{1}$ Clinic of Physical Medicine and Rehabilitation, University of Health Sciences Turkey, Ankara City Hospital, Ankara, Turkey \\ ${ }^{2}$ Clinic of Physical Medicine and Rehabilitation, Gülhane Training and Reseach Hospital, Ankara, Turkey \\ ${ }^{3}$ Department of Pathology, University of Hacettepe Faculty of Medicine, Ankara, Turkey
}

\begin{abstract}
Stewart-Treves syndrome (STS) is an angiosarcoma that usually develop in an extremity with longstanding lymphedema. Most affected patients have a history of breast cancer treated with radical mastectomy. Here, we report a case of STS with breast cancer-related lymphedema (BCRL) for a period of seven years. A 56-year-old woman presented with chronic lymphedema of the left arm. Nine years previously she had modified radical mastectomy for grade 2, invasive, ductal breast cancer. Upon physical examination, a tender, purplish lesion on the medial half of the affected arm was observed. The lesion spread rapidly with different-sized, scattered, purple-colored lesions in the affected area. A prompt skin biopsy was reported as STS. An immediate arm amputation was performed. However, a few months later she presented with new lesions on the anterior thorax and subsequent local recurrence around the scar. She received radiation-therapy. However, six months later the angiosarcoma had spread to the pelvic and upper limb area with scattered skin lesions. She had several problems during the chemotherapy and radiation-therapy, although she survived beyond 20 months.

In conclusion, STS is a rare but aggressive and important complication of BCRL. Awareness of rapidly progressing skin lesions and detailed investigation, as well as prompt surgery is necessary for patients with BCRL in order to relatively increase the survival time.

Keywords: Breast cancer, lymphedema, lymphangiosarcoma, Stewart-Treves syndrome
\end{abstract}

Cite this article as: Borman P, Yaman A, Gököz Ö. Stewart-Treves Syndrome: A Rare But Aggressive Complication of Breast Cancer-Related Lymphedema. Eur J Breast Health 2021; 17(4): 378-382.

\section{Key Points}

- $\quad$ STS is a rare but important complication of BCRL. Awareness of STS, early diagnosis, use of prompt and aggressive therapies, and close monitoring will relatively extend the survival time. Diagnostic ultrasonography may be a clinically useful imaging modality to detect possible malign transformation earlier for patients with BCRL and having suspicious skin lesions.

\section{Introduction}

Stewart-Treves syndrome (STS) is a rare and aggressive angiosarcoma developing in an extremity with longstanding lymphedema. Stewart and Treves (1) reported the first lymphangiosarcoma of the upper limb in six patients who underwent post-mastectomy in 1948. STS originates from the endothelial cells of the lymphatic and blood vessels, but the precise pathomechanism of this phenomenon remains unknown $(1,2)$. Diagnosis is made based on skin biopsy and imaging studies, and the prognosis is poor when radical surgery is not performed (3-5).

We report the case of a 56-year-old woman with STS who underwent modified radical mastectomy for breast carcinoma nine years previously and subsequent breast cancer-related lymphedema (BCRL) for seven years.

\section{Case Presentation}

In October 2016, a 56-year-old woman presented with chronic lymphedema of the left arm. She had undergone modified radical mastectomy for grade 2, invasive, ductal breast cancer and received chemotherapy, irradiation, and hormonotherapy in 2007, and had right arm edema for the seven years prior to presentation. Physical examination revealed stage 2 lymphedema with Stemmer-sign positivity. Inspection indicated a tender, purplish lesion $(1.5 \mathrm{~cm} \times 4 \mathrm{~cm}$ ) on the medial half of the affected arm (Figure 1a). She denied any trauma or infection history

This case report was presented in the $26^{\text {th }}$ World Congress of Lymphology, $25^{\text {th }}-29^{\text {th }}$ September 2017 , Barcelona, Spain as an oral presentation. 
and no history of comorbidities except hypertension. Her routine biochemical test and anticoagulation status were normal. A diagnostic ultrasonography (US) revealed areas of altered echotexture containing multiple subcutaneous, hypoechoic masses in the right inner arm with solid and cystic components (Figure $2 \mathrm{a}$ and $\mathrm{b}$ ). Due to the suspicion of metastasis, magnetic resonance imaging (MRI) was requested. The MRI showed hematoma-like, yellow-colored alterations at the edges of the patient's lesion. The lesion spread rapidly with different-sized, scattered, pink- and purple-colored lesions in the affected area (Figure 1b). A prompt skin biopsy was performed, which demonstrated large, hyperchromatic, and atypical neoplastic cells, some of which showed luminal projections. Low-power view showed extensive infiltration of the dermis by the vascular tumor. Pathology also revealed irregular, anastomosing vascular channels lined by endothelial cells exhibiting different degrees of atypia and mitotic activity, filling the dermis with cells forming luminal structures, interspersed with slit-like spaces, or small nests. Immunohistochemical staining for cluster of differentiation (CD) 31 and CD34 was positive, and pancytokeratin showed no immunoreactivity, indicating angiosarcoma and eliminating cutaneous metastases (Figures 3a, b and c). MRI indicated dermal diffuse thickening with subcutaneous enhanced-contrast nodular components, similar to the US findings (Figures $4 \mathrm{a}$ and b). Based on these results, STS was diagnosed.

Total abdominal US and chest X-ray were normal. Consultation with oncologist and orthopedic surgeon resulted in a planned, immediate, forequarter amputation. Surgical margins were found to be clear of tumor with a more than $3 \mathrm{~cm}$-cuff of tumor-free tissue. The clavicular bone biopsy appeared normal without any obvious evidence of local infiltration or metastatic seeding. During the follow-up visit of the patient, two months after the operation, the amputation scar had healed clearly (Figure 5) but she had phantom limb pain. Thus pregabalin, $150 \mathrm{mg}$ twice daily, was prescribed for phantom pain.

At the six week control visit, a new lesion on the anterior thorax was detected (Figure 6). The performed biopsy again revealed

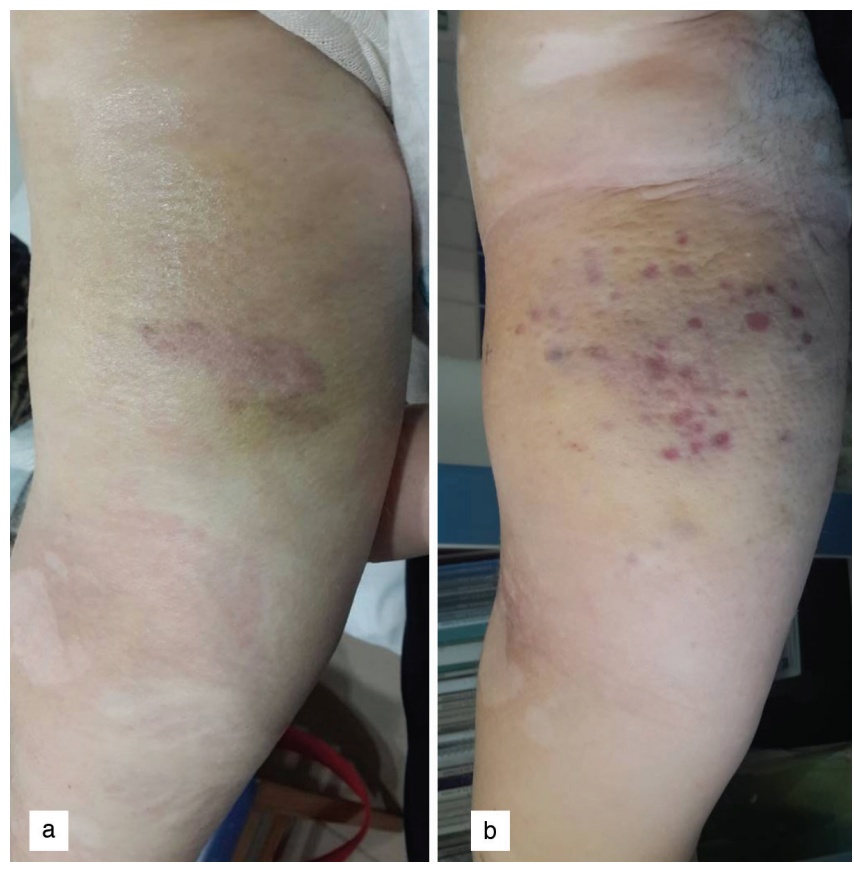

Figure 1. a, b. Purplish lesion that spread rapidly with differentsized, scattered, pink and purple-colored lesions on the right upper extremity angiosarcoma. A wide surgical excision of the tumor was performed on the anterior thorax (Figure 7). Two months later, new lesions around the scar of the thoracic mass surgery and the left axilla were detected (Figures $8 \mathrm{a}$ and b). Punch biopsy revealed further recurrence of angiosarcoma. Therefore, radiation-therapy was performed on the metastatic areas. Three months later she reported pain and similar lesions over her right hip and upper leg and attended the oncology and dermatology units (Figures 9). Pelvic bone metastasis was found on positron emission tomography scan. The management decision was palliative chemotherapy. However, during the third cycle of chemotherapy, she visited the emergency department with fever, dyspnea, cough, and breathlessness. She had severe pneumonia, resistant to multiple therapies, and was hospitalized in the intensive care unit for three weeks. After resolution of symptoms and findings,
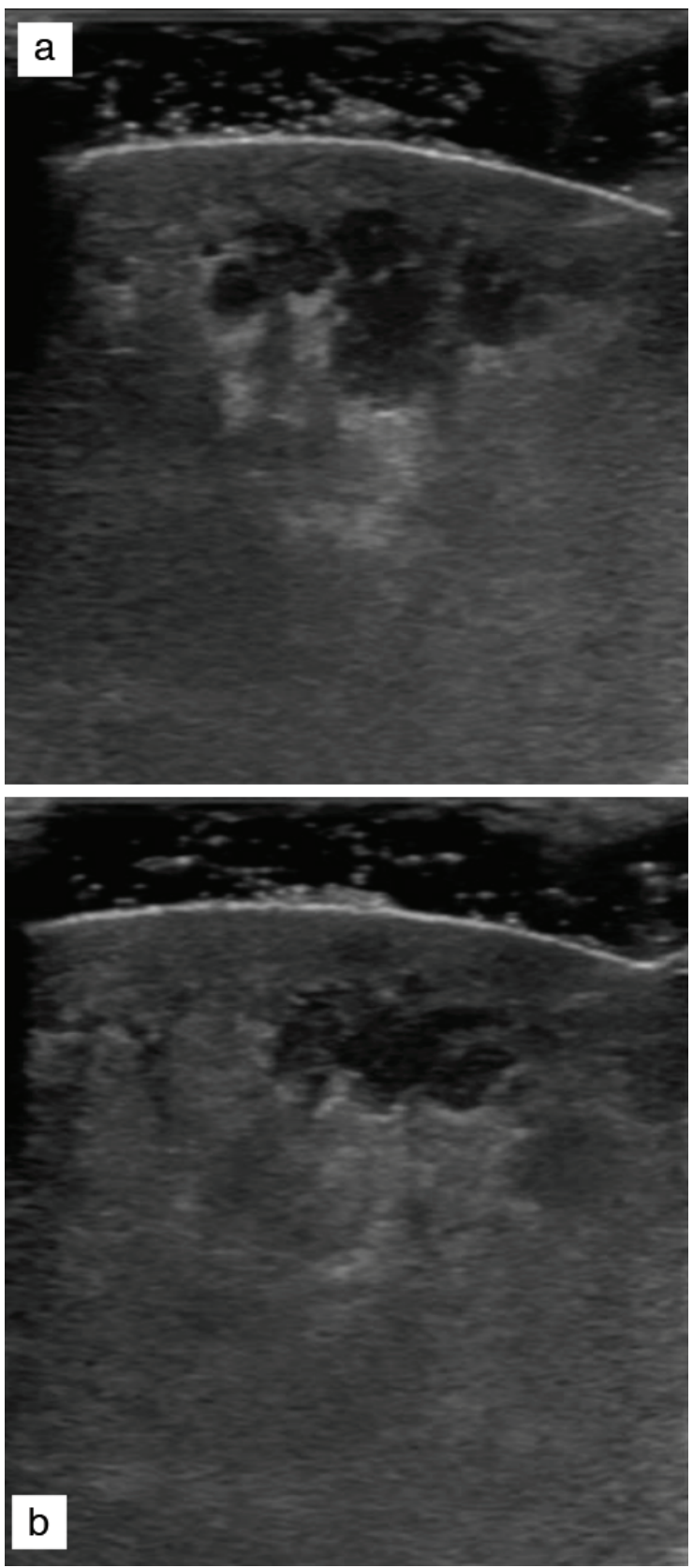

Figure 2. a, b. US revealed multiple masses in the right inner arm with solid and cystic components appearing as subcutaneous irregular hypoechoeic lesions

US: Ultrasonography 
she was discharged with palliative suggestions. A few weeks later, she received radiation-therapy for the pelvic metastatic area and chemotherapy was initiated again. On her final visit, three months after chemotherapy completeion, she had severe pain in the lower back and legs. She was placed on palliative pain control but subsequently developed urinary tract infection and was lost due to sepsis.

\section{Discussion and Conclusion}

STS is a rare but deadly angiosarcoma that develops due to chronic lymphatic obstruction; STS may be either primary or secondary (1-10), and affects an estimated $0.07 \%-0.45 \%$ of patients who survive longer than five years after radical mastectomy $(7,10-12)$. Causative factors including radiation-therapy, immunodeficiency, and an association between STS and cardiovascular conditions have previously been reported (11), but the underlying pathophysiology is still uncertain $(2,13)$. The period between the onset of lymphedema and the appearance of STS lesions varies between 5-11 years. The tumor is characterized by multiple purplish, painless, macular lesions, which may be dismissed, and then develops into a plaque or nodule (3, 7, 13-15). The diagnosis depends on histopathological findings but imaging-modalities can also be helpful $(3,4,15)$. Histopathological findings may vary among cases but are commonly reported as irregular and anastomosed endothelial tissue in the most-differentiated areas, as well as atypical epitheloid and spindle masses in less-differentiated areas. Immunopathological studies indicate positive staining for endothelial cell markers comprising laminin and antibodies against CD31 and CD34 are positive. Absence of epithelial differentiation markers, such as cytokeratin, helps in the elimination of cutaneous metastases of breast cancer $(2,4,11,13,15)$.

Our patient had right, modified, radical mastectomy with resection of 26 axillary-nodes and postoperative radiation nine years previously, and lymphedema had been evident for more than seven years. However, our patient was relatively young, and the survival period was relatively longer to identify the aggressive nature of the tumor and high-risk recurrence affecting different areas and responsiveness to radical therapies. In our case US was performed and revealed similar lesions to
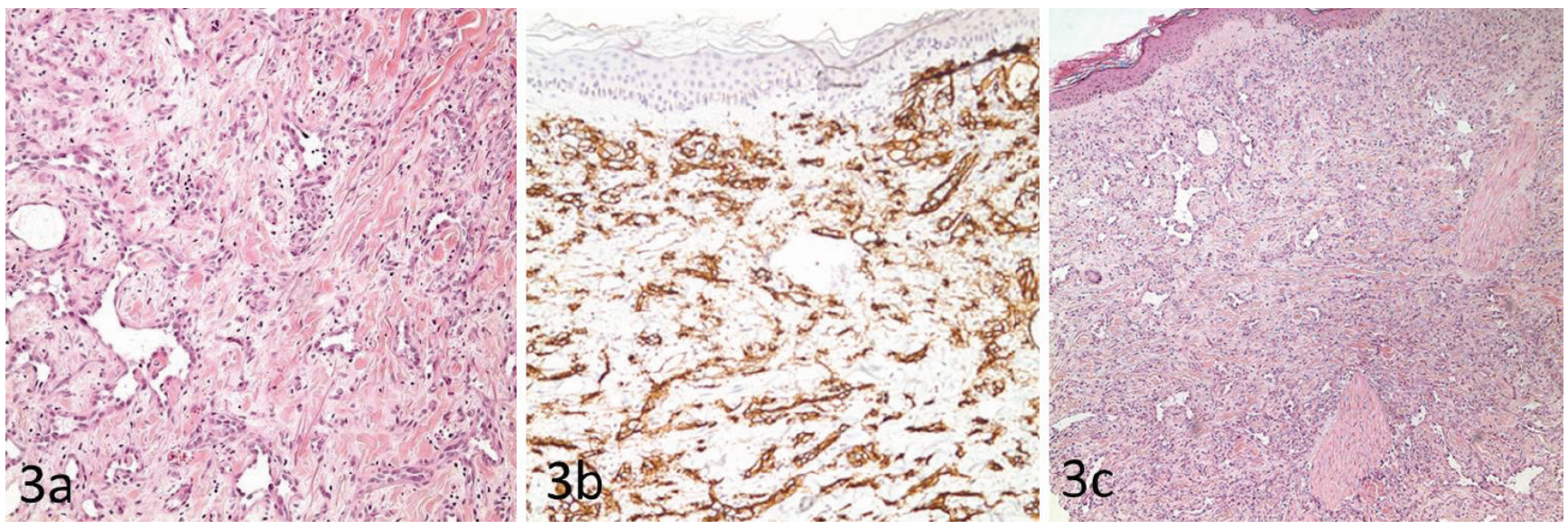

Figure 3. a, b, c. Histological examination revealed irregular anastomosing vascular channels lined with endothelial cells exhibiting different degrees of atypia and mitotic activity, filling the dermis with cells forming luminal structures, slit-like spaces, or small nests. Immunohistochemical staining for CD31 and CD34 was positive

CD: Cluster of differentiation
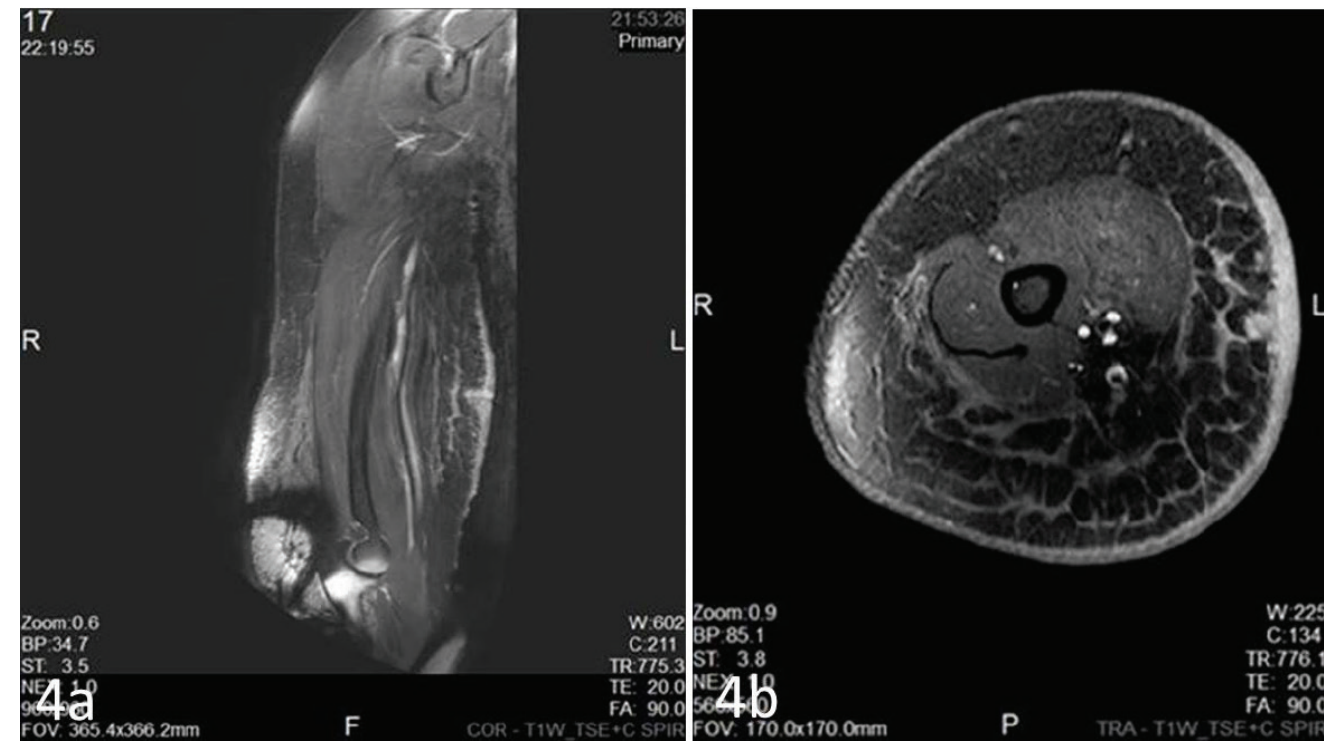

Figure 4. a, b. MRI indicated dermal diffuse thickness with subcutaneous enhanced-contrasting nodular components 


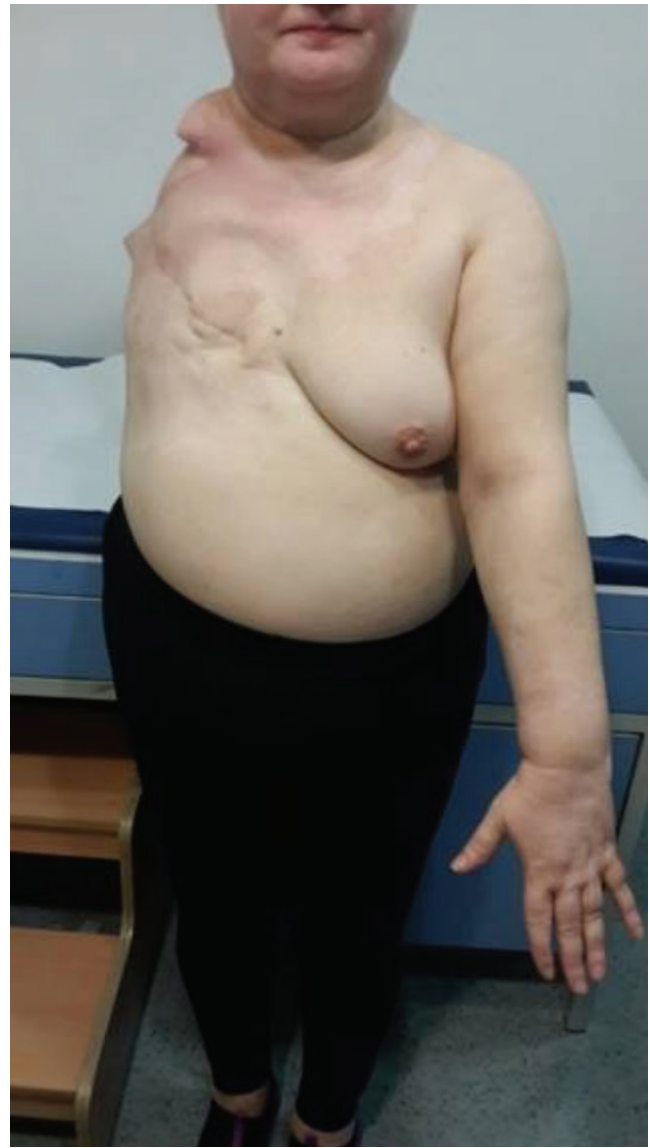

Figure 5. Amputation scar after 2 months

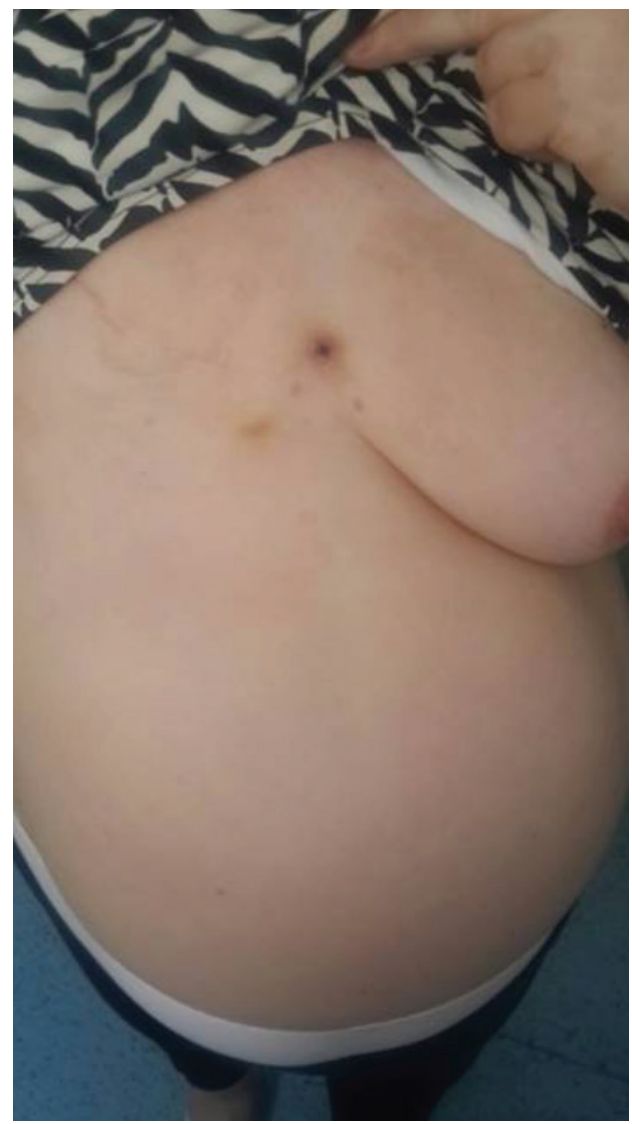

Figure 6. New lesion on the anterior thorax

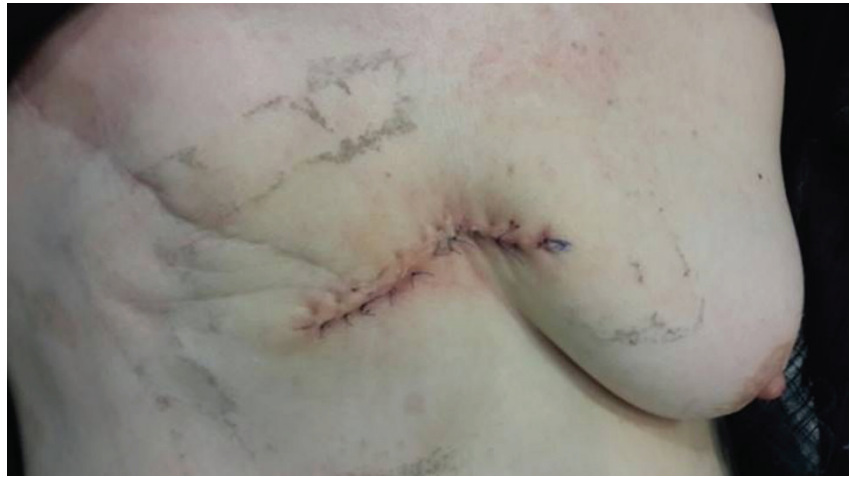

Figure 7. Wide surgical excision of the tumor on the anterior thorax

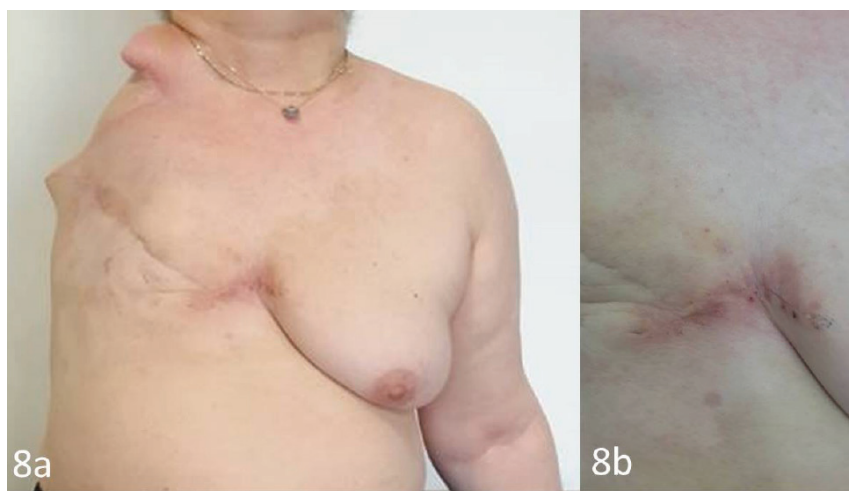

Figure 8. a, b. New lesions around the scar of the thoracic mass surgery and on the left axilla, three months after the second surgery

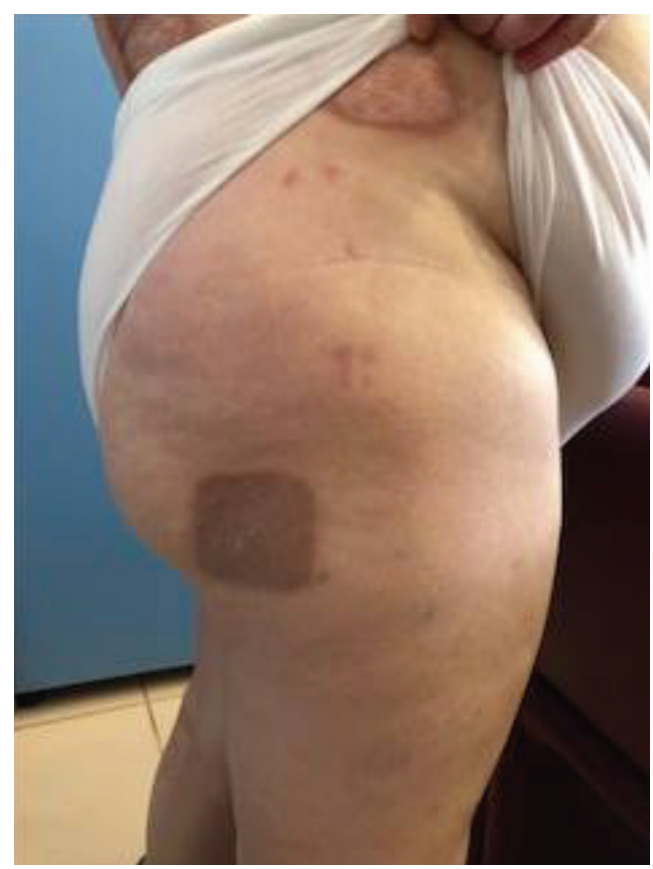

Figure 9. New skin lesions in gluteal area and right upper leg in which punch biopsies were taken

those reported on MRI findings. In recent years, US has been widely used in patients with lymphedema for differential diagnosis to monitor effects of treatments (16). Angiosarcomas have variable features on US examination, such as well-circumscribed or poorly marginated hypoechoic or hyperechoic masses, and US was previously used to 
visualize these lesions (17). We suggest diagnostic US for evaluation of STS skin lesions and early diagnosis, as a practical, easy, and affordable method. However, further studies are needed to confirm the diagnostic value and advantages of US over MRI or computed tomography in such lesions.

STS has poor prognosis, and due to its rarity and high rate of local recurrence and metastatic disease, no standardized therapy is recommended $(2,3,7,5,15)$. As the presentation of the tumor may be confused with traumatic ecchymosis or benign vascular lesions, awareness of this condition is crucial. Our patient had a relatively short duration between the development of lymphedema and presentation of angiosarcoma. After prompt STS diagnosis, early radical amputation was performed because of the local extent of the tumor and the likelihood of it being very aggressive. Unfortunatly, the tumor was so aggressive that it spread to the anterior thorax just a few months later, and local recurrences and bone metastasis were observed within a few months. Our case also demonstrates that even prompt amputation does not guarantee prevention of local recurrence, and recurrences may be common after wide surgical resections. With multi-modal and aggressive management, she managed to survive beyond 20 months.

In conclusion, STS is a rare but important complication of BCRL. Awareness, early diagnosis, prompt and aggressive therapies, and close monitoring will relatively improve the duration of survival time, as seen in our patient. US may be a clinically useful imaging modality for earlier detection of possible malign transformation in patients with BCRL having suspicious skin lesions.

Informed Consent: It was obtained.

Peer-review: Externally peer-reviewed.

\section{Author Contributions}

Conception: P.B.; Design: A.Y.; Supervision: P.B.; Materials: A.Y.; Data Collection and/or Processing: A.Y.; Analysis and/or Interpretation: Ö.G.; Literature Search: P.B.; Writing: P.B.

Conflict of Interest: The authors have no conflicts of interest to declare.

Financial Disclosure: The authors declared that this study has received no financial support

\section{References}

1. Stewart FW, Treves N. Lymphangiosarcoma in postmastectomy lymphoedema: a report of six cases in elephantiasis chirurgica. Cancer 1948; 1: 64-81. (PMID: 18867440) [Crossref]

2. Sharma A, Schwartz RA. Stewart Treves syndrome: pathogenesis and management. J Am Acad Dermatol 2012; 67: 1342-1348. (PMID: 22682884) [Crossref]

3. Berebichez-Fridman R, Deutsch YE, Joyal TM, Molvera PB, Benedetto PW, et al. Stewart-Treves Syndrome: a case report and review of the literature. Case Rep Oncol 2016; 9: 205-211. (PMID: 27099606) [Crossref]
4. Li B, Wang Z. Stewart-Treves syndrome: magnetic resonance imaging data compared with pathological results from a single center. Oncol Lett 2018; 15: 1113-1118. (PMID: 29391898) [Crossref]

5. Farzaliyev F, Hamacher R, Steinau HU, Bertram S, Podleska LE. Secondary angiosarcoma: a fatal complication of chronic lymphedema. J Surg Oncol 2020; 121: 85-90. (PMID: 31236970) [Crossref]

6. Lee R, Saardi KM, Schwartz RA. Lymphedema related angiogenic tumors and other malignancies. Clin Dermatol 2014; 32: 616-620. (PMID: 25160102) [Crossref]

7. Cui L, Zhang J, Zhang X, Chang H, Qu C, Zhang J, et al. Angiosarcoma (Stewart-Treves syndrome)in postmastectomy patients: report of 10 cases and review of the literature. Int J Clin Exp Pathol 2015; 8: 11108-11115. (PMID: 26617830) [Crossref]

8. Tabareau-Delalande F, de Muret A, Miquelestorena-Standley E, Decouvelaere AV, de Pinieux G. Cutaneous epitheloid clear cells angiosarcoma in a young woman with congenital lymphedema. Case Rep Pathol 2013; 2013: 931973. (PMID: 24078891) [Crossref]

9. Cabral ANF, Rocha RH, Amaral ACVD, Medeiros KB, Nogueira PSE, Diniz LM. Cutaneous angiosarcoma: report of three different and typical cases admitted in a unique dermatology clinic. An Bras Dermatol 2017; 92: 235-238. (PMID: 28538886) [Crossref]

10. Taşdemir A, Karaman H, Ünal D, Mutlu H. Stewart-Treves Syndrome after bilateral mastectomy and radiotherapy for breast carcinoma: Case report. J Breast Health 2015; 11: 92-94. (PMID: 28331699) [Crossref]

11. Felmerer G, Dowlatshahi AS, Stark GB, Földi E, Földi M, Ahls MG, et al. Lymphangiosarcoma: is Stewart-Treves Syndrome a preventable condition? Lymphat Res Biol 2016; 14: 35-39. (PMID: 26584023) [Crossref]

12. Alan S, Aktas H, Ersoy ÖF, Aktümen A, Erol H. Stewart-Treves syndrome in a woman with mastectomy. J Clin Diagn Res 2016; 10: WD01-WD02. (PMID: 27042566) [Crossref]

13. Wang LL, Cui LF, Gao Y, Jiang ZC. Clinicopathologic features of StewartTreves syndrome. Int J Clin Exp Pathol 2019; 12: 680-688. (PMID: 31933875) [Crossref]

14. Pegas Pereira ES, Siqueira DM, Moraes ET, dos Santos MAS. Stewart Treves Syndrome. An Bras Dermatol 2015; (Suppl 1):S229-S231. (PMID: 26312725) [Crossref]

15. Mesli SN, Ghouali AK, Benemara F, Taleb FA, Tahraoui H, Abi-Ayad C. Stewart-Treves syndrome involving chronic lymphedema after mastectomy of breast cancer. Case Rep Surg 2017;2017:4056459. (PMID: 28280645) [Crossref]

16. Mander A, Venossi S, Menegatti E, Byung-Boong L, Neuhardt D, et al. Upper limb secondary lymphedema ultrasonographic mapping and characterization. Int Angiol 2019; 38: 334-342. (PMID: 31203598) [Crossref]

17. Taghipour-Zahir S, Sefiddrokh-Sharahjin N, Rahmani K. Primary breast angiosarcoma: pathological and radiological diagnosis. Malays J Med Sci 2014; 21: 66-70. (PMID: 25977636) [Crossref] 